\title{
Wavelet estimation using the Multi-Layer Perceptron Neural Network algorithm
}

\section{Lorena da S. O. Santos*, INCT-GP/UFBA, Jonh B. Lemos, INCT-GP/UFBA and Alexsandro G. Cerqueira, INCT-GP/UFBA}

Copyright 2021, SBGf - Sociedade Brasileira de Geofísica.

This paper was prepared for presentation during the $17^{\text {th }}$ International Congress of the Brazilian Geophysical Society held in Rio de Janeiro, Brazil, $16-19$ August 2021.

Contents of this paper were reviewed by the Technical Committee of the $17^{\text {th }}$ International Congress of the Brazilian Geophysical Society and do not necessarily represent any position of the SBGf, its officers or members. Electronic reproduction or storage of any part of this paper for commercial purposes without the written consent of the Brazilian Geophysical Society is prohibited.

\section{Abstract}

\section{Summary}

Wavelet estimation is a fundamental step in the seismic reflection method, especially in well tie and seismic inversion. Overall, we can split the methods for wavelet estimation into two: deterministic and stochastic. Deterministic approaches are the ones in which the wavelet obtention is from well ties using borehole measurements. On the other hand, stochastic methods use seismic reflection data to estimate the wavelet (Bacon and Simm, 2014).

Artificial neural networks (ANN's) are computational models based on biological neuron activity. A multi-layer perceptron network (MLP) is a network composed of input (Silva et al., 2016), hidden, and output layers that can be employed to solve several geophysical problems.

To implement a neural network able to deliver a suitable wavelet estimation, we have created one hundred thousand synthetic seismic models containing layers with different values of thickness, velocity, and density. Density values ranged between 2 and $3.5 \mathrm{~kg} / \mathrm{m}^{3}$, and the velocity between 1600 and $3500 \mathrm{~m} / \mathrm{s}$. Velocity values increase gradually with depths. The reflectivity coefficient models extracted from these profiles were convolved with a wavelet to generate the synthetic seismic trace. The wavelets used in this work were: Ricker, Gabor, and Ormsby, their frequency content ranged from 5 to $60 \mathrm{~Hz}$, and they were randomly picked by the algorithm. After convolving the model with the wavelets, we added some noise to the synthetic data.

The synthetic seismic traces were the input of the multioutput MLP network, and their wavelets were the target. To implement the MLP network, the Keras open-source library in the python language was used (Chollet and others, 2015).

\section{Results}

To carry out the experiments, we split the dataset into two subsets: $75 \%$ of the dataset was used to train the neural network, and $25 \%$ to evaluate the learning rate of the ANN. The cost function adopted to train the neural network was the logarithm of the hyperbolic cosine (logcosh) and the train and test dataset cosine similarity were respectively 0.995 and 0.973 .

Figure 1 shows some wavelets, and their prediction through the neural network model. Visually, the wavelets seem remarkably similar to the original wavelet, and most of the model predictions displayed a high Pearson correlation, bigger than 0.9 when compared to their target wavelets.
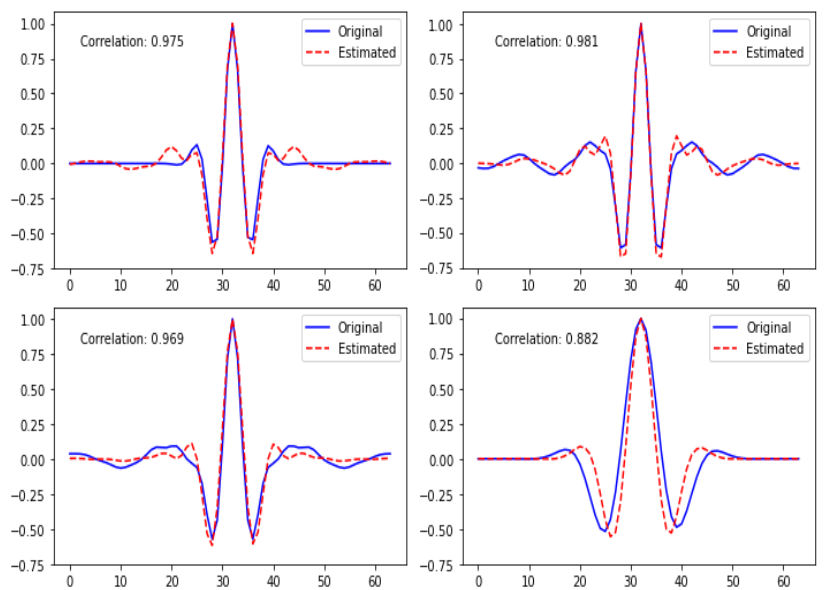

Figure 1 - Target wavelet (blue line), wavelet predicted by the ANN algorithm (red dashed line), and the Pearson correlation between them.

\section{Conclusions}

The logarithm of the hyperbolic cosine was used as the cost function of the neural network. The MLP was able to predict the wavelets with high accuracy. The results also displayed a good correlation between the original and the wavelet estimated by the algorithm.

\section{References}

Simm, R, and M Bacon. Seismic amplitude: an interpreter's handbook. Cambridge New York: Cambridge University Press, 2014. Print.

Silva, I., Spatti, D., Flauzino, R., Liboni, L. \& Alves, S. (2016). Artificial neural networks: a practical course. Switzerland: Springer.

Chollet, F., \& others. (2015). Keras. https://keras.io. 